\title{
Impact of ureteral stenting prior to ureterorenoscopy on stone-free rates and complications
}

\author{
P. P. Lumma $\cdot$ P. Schneider $\cdot$ A. Strauss $\cdot$ \\ K. D. Plothe $\cdot$ P. Thelen $\cdot$ R. H. Ringert $\cdot$ \\ H. Loertzer
}

Received: 5 July 2011/ Accepted: 12 October 2011/Published online: 29 October 2011

(c) The Author(s) 2011. This article is published with open access at Springerlink.com

\begin{abstract}
Objective To date, only few studies have evaluated the impact of ureteral stenting prior to ureterorenoscopy. This study is to clarify the role of preoperative ureteral stenting in the treatment for ureteral stones.

Methods We retrospectively reviewed 550 ureterorenoscopies from 1998 to 2008. Patients were classified into two groups depending on whether they had a stent placed before URS. Baseline characteristics of patients and stone properties, stone-free rates, complications, and operation times were compared between both groups. Subanalysis was performed regarding stone localization. We retrospectively reviewed data from patient documentation, $\mathrm{X}$-ray imagery, intravenous urography, and operation reports.

Results Baseline characteristics of patients were similar in both groups. The majority of patients underwent stent placement before the ureteroscopic stone treatment $(88.4 \%)$. The mean operation time in the prestented group was longer (43.3 vs. $38.4 \mathrm{~min}$ ). Stone-free rate of patients with stent was $72.2 \%$, compared to $59.4 \%$ without preoperative stenting. The rate of minor complications was $4.7 \%$ with stent versus $9.4 \%$ without stent, major complications $0.6 \%$ versus $1.6 \%$, respectively. Patients with distal ureter stones had similar stone-free rates regardless of a stent placement $(90.1 \%$ with stent vs. $87.6 \%$ without), and no
\end{abstract}

P. P. Lumma - P. Schneider · A. Strauss ·

K. D. Plothe · P. Thelen · R. H. Ringert · H. Loertzer $(\square)$

Department of Urology, University Medical Center Göttingen,

Georg-August-University, Robert-Koch-Str. 40,

37075 Göttingen, Germany

e-mail: hagen.loertzer@med.uni-goettingen.de

URL: http://www.urologie.uni-goettingen.de difference in complication rates was observed $(3.5 \%$ with stent vs. $3.1 \%$ without), respectively.

Conclusions Stent placement prior to ureteroscopic stone treatment in distal ureter is not reasonable and does not considerably improve stone-free rates.

Keywords Ureterorenoscopy · Urolithiasis · Complication after ureterorenoscopy $\cdot$ Stone-free rates . Preoperative ureteral stenting

\begin{tabular}{|c|c|}
\hline \multicolumn{2}{|c|}{ Abbreviations } \\
\hline $\mathrm{Ch}$ & $\begin{array}{l}\text { Charrière, diameter of urologic instruments, } \\
\text { catheters, and endoscopes. } 1 \mathrm{Ch}=1\end{array}$ \\
\hline & French $=1 / 3 \mathrm{~mm}$ \\
\hline DJ & Double-J ureteral stent \\
\hline ESWL & Extracorporal shock wave lithotripsy \\
\hline IVU & Intravenous urogram \\
\hline URS & Ureterorenoscopy \\
\hline
\end{tabular}

\section{Introduction}

The role of stent placement after ureterorenoscopic stone treatment has been evaluated by many studies according to which routine stenting after uncomplicated ureteroscopic stone procedure is not necessary. Associated complications such as stent migration, breakage, encrustation, urinary tract infection, and obstruction were observed. Furthermore, a secondary cystoscopy was required for stone removal in many cases. Clear indications exist for postoperative stenting after ureterorenoscopy. These are among others solitary kidney, renal insufficiency, ureteral injury, stricture, or a large residual stone burden $[3,5,7]$. 
Table 1 Patient characteristics

\begin{tabular}{llll}
\hline & DJ before URS & No DJ before URS & Total \\
\hline Procedures & 486 & 64 & 550 \\
Age (years) & 50.7 & 49.9 & 50.6 \\
Gender & & & \\
Men & 336 & 49 & 385 \\
Women & 150 & 15 & 165 \\
Stone size & & & \\
Max. diameter (mm) & 5.3 & 5.6 & 5.3 \\
$\leq 5$ mm & 292 & 31 & 323 \\
$>5$ mm $\leq 10$ mm & 167 & 29 & 196 \\
$>10$ mm & 27 & 4 & 31 \\
Stone location & & & \\
Renal pelvis & 95 & 8 & 103 \\
Prox. ureter & 59 & 11 & 70 \\
Mid. ureter & 71 & 10 & 81 \\
Distal. ureter & 172 & 32 & 204 \\
Multiple & 89 & 3 & 92 \\
\hline
\end{tabular}

However, only few studies have been published that focus on preoperative double-j stenting. Although this technique is frequently used, little is known about its indications and results. Hence, the purpose of this study is to clarify the role of prestenting for the procedures' results.

Therefore, we reviewed the data of several hundred ureterorenoscopic stone treatments.

\section{Patients and methods}

We retrospectively reviewed 550 ureterorenoscopic procedures (URS) from 474 individual patients between January 1998 and December 2008. These patients were classified into two groups depending on whether they had a stent placed before URS. One group of patients had received preoperative stent placement (group 1), whereas the other had not (group 2). Group 1 received preoperative stents because clinical evidence of stones or persistent renal colics existed before the URS. Group 2 had received URS as a diagnostic procedure to further examine tumor suspicion, suspicion of abnormal ureter lumina, ureter structure, or hematuria located in the ureter. With all included patients in group 2, stones were incidentally found; meanwhile, no other abnormality was detected in the URS. All URS were performed or supervised by specialists in the Department of Urology, University of Goettingen, Germany.

Stones visible in X-ray imaging or intravenous urography were measured for their linear diameter, and stone location was noted. Both determined the choice of the ureterorenoscope. We used semirigid (OES PRO ${ }^{\circledR}$,
Olympus) and flexible ureterorenoscopes (URF-P5 ${ }^{\circledR}$ and DUR-8 Elite ${ }^{\circledR}$, Olympus).

Preoperatively as a rule $6 \mathrm{Ch}$, double-j ureteral stents were used. Depending on the patient's size, the stents were between 26 and $30 \mathrm{~cm}$ long. All stents were placed using a $21 \mathrm{Ch}$ endoscope (Olympus). Anesthetization was done facultatively with Midazolam (Dormicum ${ }^{\circledR}$ ) or Piritramid $\left(\right.$ Dipidolor $\left.^{\circledR}\right)$. Nephrostomy was necessary when contrast medium instilled into the ureter showed obstructing ureteral calculi. All stents were removed at the beginning of the ureteroscopic procedure before stone removal.

We retrospectively reviewed data from patient documentation, X-ray imagery, intravenous urography, and operation reports. The statistics were done with "Open Office Calc" (version 3.1) and "R" (version 2.9.2).

\section{Results}

Both groups (with and without stent placement) had similar features concerning the patients' age, gender ratio, and diameter of stones (Table 1). More distal ureter stones and stones in a single urinary tract location were observed in the unstented group. The most striking difference between both groups was the total amount of performed procedures. Patients underwent stent placement prior to stone treatment in $88.4 \%$ of all 550 ureterorenoscopies.

A successful stone-free status was achieved when all endoscopically or radiographically visible stone fragments had been removed completely. The operation time was extended by $4.9 \mathrm{~min}$ when prestented patients were treated (43.3 vs. $38.4 \mathrm{~min}$ ). Patients who underwent stent placement had a higher stone-free rate than nonstented patients $(72.2 \%, n=351$ vs. $59.4 \%, n=38)$. Stented patients had less complications than patients without preoperative stent placement (minor complications: $4.7 \%, n=23$ vs. 9.4\%, $n=6$, major complications: $0.6 \%, n=3$ vs. $1.6 \%, n=1$ ) (Fig. 1). Perforations of ureter or renal pelvis were defined as minor complications in this study together with hematuria and obstructive urinary flow disorders. Minor complications were treated with stent placement at the end of the procedure. Major complications were ureter avulsion, urosepsis, and bleeding with need for blood transfusion. Major complications required immediate and extensive intervention in contrast to minor complications.

Considerably, more ureter perforations occurred in the non-stented patient group. 13 ureteral perforations were observed after 486 ureterorenoscopies with preoperative stent placement (2.7\%), compared to 6 perforations after 64 treatments without stenting $(9.4 \%)$ (Table 2$)$.

One or more stones in a single urinary tract location were treated in 458 ureterorenoscopies (Fig. 2), whereas stones in different locations of the urinary tract were 
Fig. 1 All procedures to difference with DJ or without DJ before URS
QDJ before URS $\square$ no DJ before URS

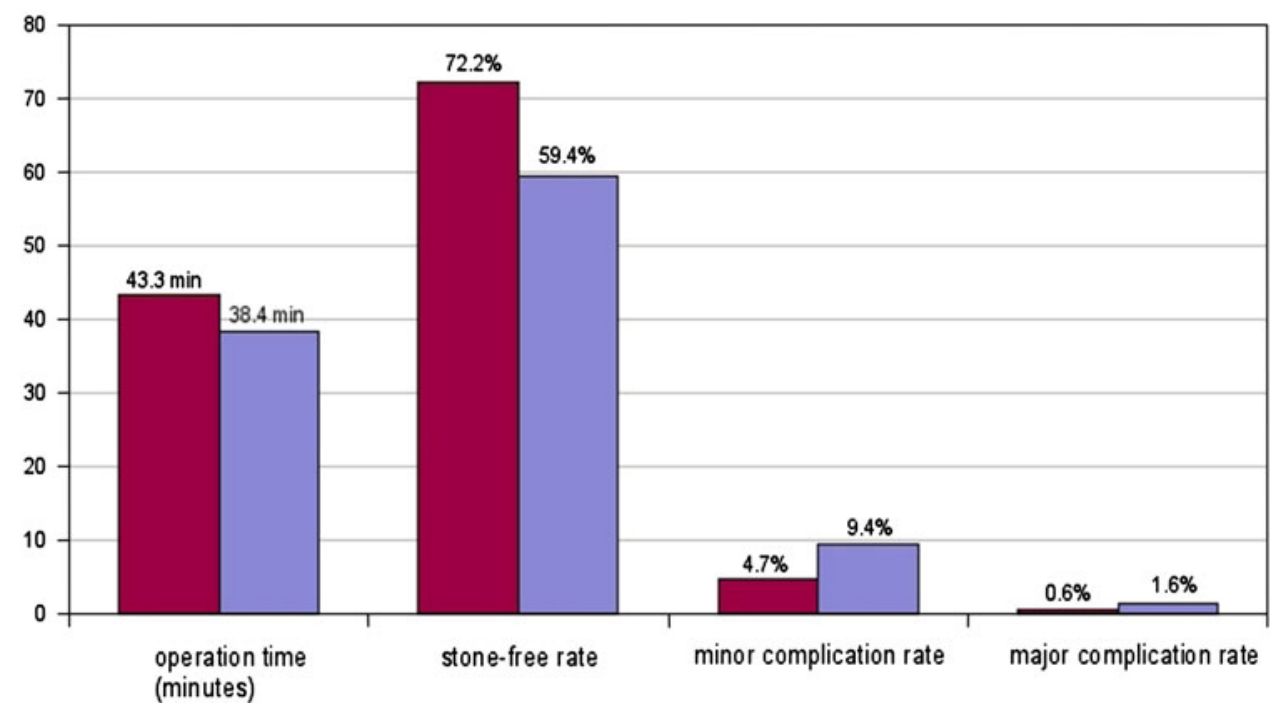

Table 2 Complication all procedures to difference with DJ or without DJ before URS

\begin{tabular}{lllr}
\hline & $\begin{array}{l}\text { DJ before } \\
\text { URS }\end{array}$ & $\begin{array}{l}\text { No DJ before } \\
\text { URS }\end{array}$ & Total \\
\hline Ureter perforation & 13 & 6 & 19 \\
Renal pelvis perforation & 10 & - & 10 \\
Ureter avulsion & 1 & - & 1 \\
Urosepsis & 1 & - & 1 \\
$\begin{array}{l}\text { Bleeding (with } \\
\text { transfusion) }\end{array}$ & 1 & 1 & 2 \\
\hline
\end{tabular}

treated in 92 procedures. Prestented patients had an improved stone-free rate compared to non-stented patients when the stone was located in the proximal urinary tract (mid/proximal ureter or renal pelvis) $(67.1 \%, n=150$ vs. $34.5 \%, n=10$ ). Less complications occurred in the proximal urinary tract when patients had undergone preoperative stent placement $(7.1 \%, n=16$ vs. $17.2 \%, n=5)$. When distal ureter calculi were treated, the stone-free rate was nearly the same in both groups $(90.1 \%, n=154$ vs. $87.5 \%, n=28$ ), whereas the rate of complications was slightly increased in the group of patients which received a
Fig. 2 Preoperative DJstending in distal and proximal urinary tract
DDJ before URS $\square$ no DJ before URS

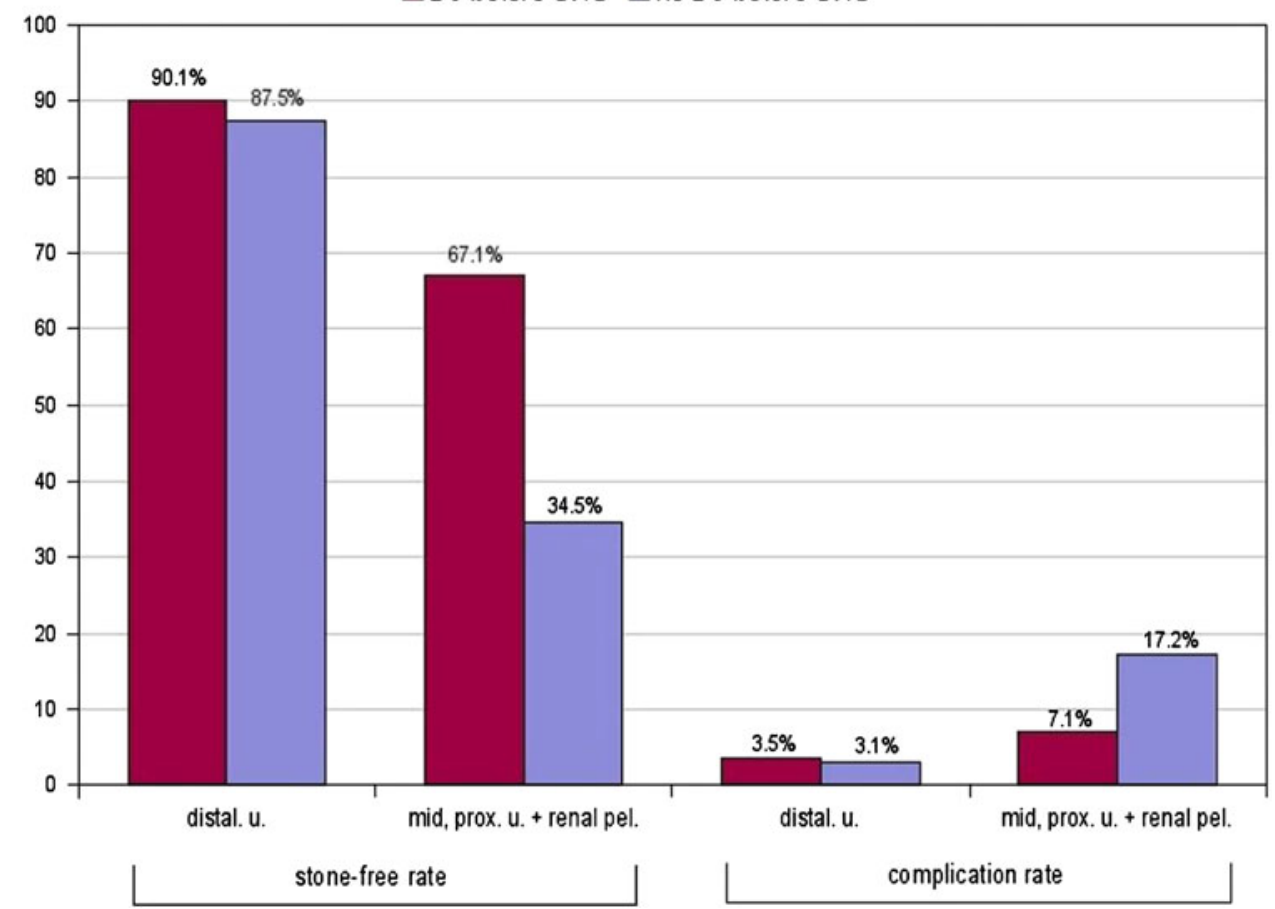


stent prior to ureterorenoscopy $(3.5 \%, n=6$ vs. $3.1 \%$, $n=1$ ) (Fig. 2).

\section{Discussion}

Although it is a frequently used technique, only few studies have evaluated the role of ureteral stenting before ureterorenoscopic stone treatment. Therefore, questions about its impact on procedures' results and correct indications remain unsolved. The purpose of this analysis was to provide answers concerning results and complications of prestenting.

The operation time of prestented patients was extended compared to patients without stent placement before ureterorenoscopy by $4.9 \mathrm{~min}$.

In our opinion, stent extraction prior to ureterorenoscopic stone treatment caused the difference in operation time between both groups. No publications could be found concerning this issue. Chander and colleagues reported that prestenting had no effect on the operation time of retroperitoneal laparoscopic pyelolithotomy. This result was not significant and probably not relevant for the results of ureterorenoscopic stone treatment [1].

The impact of stent placement prior to ureterorenoscopy on the results of ureteral stone treatment has provoked controversial discussion. Corcoran et al. and Hubert and Palmer reviewed the impact of prestenting on the results of ureterorenoscopy in children ( $<18$ years). Corcoran et al. reported that prestenting reduced additional treatments and yielded a low complication rate after primary ureterorenoscopy had failed. Hubert and Palmer were able to avoid active dilatation of the ureter in all cases after stent placement prior to ureteroscopy [2, 4]. Rubenstein et al., Shields et al., and Unsal et al. analyzed the impact of preoperative stenting before ureterorenoscopy on the outcome in adults. All three publications come to the conclusion that preoperative stenting is optional and not obligatory. Rubenstein et al. and Shields et al. point out that stent placement can result in better stone-free rates [8-10].

Our results show that preoperative stenting considerably improved the stone-free rates with proximal located stones (mid/proximal ureter and renal pelvis). In contrast, the stone-free rate of stones treated in distal ureter was not considerably increased after preoperative stent placement (Fig. 2).

Rubenstein and colleagues reviewed 115 ureterorenoscopic treatments of ureter and renal pelvis stones. The stone-free rate of prestented patients was significantly higher than that of patients without stent [8]. Shields et al. reported similar results of 259 ureteroscopies for ureter stones. Patients who had received a stent prior to ureterorenoscopy had an increased stone-free rate although this result was not significant [9]. Musa analyzed the impact of prestenting on the outcome of $120 \mathrm{ESWL}$ treatments. In this study, patients with stent prior to ureterorenoscopy had no improvement of stone-free rates [6]. These findings are in agreement with our observations. We found a considerably higher stone-free rate of prestented patients compared to patients without stent placement when the stone was located in mid/proximal ureter or renal pelvis. However, when distal ureter stones were treated, both groups showed nearly equal stone-free rates. To our knowledge, there is no report available to discuss this issue. However, Shields et al. placed stents more frequently prior to ureterorenoscopy when stones in proximal ureter were treated $(51.6 \%$ stented with distal ureter stones, $79.2 \%$ stented with stones in renal pelvis). The resulting considerable stone-free rate then was $83.3 \%$ for stones in renal pelvis [9].

In our study, prestented patients had fewer complications than patients who did not receive a stent before stone treatment (minor complications $4.7 \%$ vs. 9.4\%, major complications $0.6 \%$ vs. $1.6 \%$ ) (Fig. 1). Especially, ureter perforations occurred more frequently in the non-stented group (2.7\% vs. $9.4 \%$ ). Analysis of the subgroups revealed that patients without stent who were treated for stones in $\mathrm{mid} /$ proximal ureter and renal pelvis had more complications than stented patients $(17.2 \%$ vs. $7.1 \%)$. However, when distal ureter stones were treated, there was no difference between both groups regarding the complication rate $3.1 \%$ in the nonstented group vs. $3.5 \%$ in the prestented group, respectively) (Fig. 2).

Rubenstein et al. reported hydronephrosis and ureter stricture as complications in one case in both groups (36 patients stented and 97 patients not stented prior to ureterorenoscopy). In addition, patients without stent had a subcapsular hematoma in one case and delayed voiding revealed on IVU in two cases. On a generally low complication level, there was no significant difference in the complication rate between both groups [8]. Hubert and Palmer analyzed the effect of preoperative stenting on the results of ureteroscopic stone treatment in infants. The authors reported no complications at all in both groups, stented and not [4]. However, no further subanalysis was performed regarding stone location and complications. To our knowledge, there is no report available discussing this issue.

\section{Conclusion}

We reviewed literature and did not find any association between preoperative stenting and an increased complication rate. Prestented patients who were treated for mid/ 
proximal ureter stones had improved stone-free rates and less complications compared to patients without stent. In contrast to this placing, a stent prior to the treatment for distal stones by URS did not result in a benefit for the treated patients. This leads to the conclusion that preoperative stent placement for the treatment for distal ureter stones is not reasonable.

Conflict of interest The authors declare that they have no conflict of interest.

Open Access This article is distributed under the terms of the Creative Commons Attribution Noncommercial License which permits any noncommercial use, distribution, and reproduction in any medium, provided the original author(s) and source are credited.

\section{References}

1. Chander J, Dangi AD, Gupta N et al (2010) Evaluation of the role of preoperative double-j ureteral stenting in retroperitoneal laparoscopic pyelolithotomy. Surg Endosc 24(7):1722-1726

2. Corcoran AT, Smaldone MC, Mally D et al (2008) When is prior ureteral stent placement necessary to access the upper urinary tract in prepubertal children? J Urol 180(4 Suppl):1861-1863 (discussion 1863-1864)

3. Gerber GS, Stockton BR (2006) Use of stents after ureteroscopic stone removal. J Endourol 20(6):383-385

4. Hubert KC, Palmer JS (2005) Passive dilation by ureteral stenting before ureteroscopy: eliminating the need for active dilation. J Urol 174(3):1079-1080 (discussion 1080)

5. Knoll T (2009) Arbeitskreis Harnsteine der Akademie der Deutschen Urologen, Arbeitskreis Endourologie und Steinerkrankung der Osterreichischen Gesellschaft fuer Urologie. S2 guidelines on diagnostic, therapy and metaphylaxis of urolithiasis : part 1: diagnostic and therapy. Urologe A 48(8):917-924

6. Musa AAK (2008) Use of double-j stents prior to extracorporeal shock wave lithotripsy is not beneficial: results of a prospective randomized study. Int Urol Nephrol 40(1):19-22

7. Preminger GM, Tiselius HG, Assimos DG et al (2007) Guideline for the management of ureteral calculi. J Urol 178(6):2418-2434

8. Rubenstein RA, Zhao LC, Loeb $S$ et al (2007) Prestenting improves ureteroscopic stone-free rates. J Endourol 21(11):12771280

9. Shields JM, Bird VG, Graves R et al (2009) Impact of preoperative ureteral stenting on outcome of ureteroscopic treatment for urinary lithiasis. J Urol 182(6):2768-2774

10. Unsal A, Cimentepe E, Balbay MD (2004) Routine ureteral dilatation is not necessary for ureteroscopy. Int Urol Nephrol 36(4):503-506 